\title{
Atypical Antipsychotic Drug Olanzapine Deregulates Hepatic Lipid Metabolism and Aortic Inflammation and Aggravates Atherosclerosis
}

\author{
Chia-Hui Chen ${ }^{a}$ Song-Kun Shyue ${ }^{b}$ Chiao-Po Hsur,d Tzong-Shyuan Lee ${ }^{\mathrm{a}, \mathrm{e}}$ \\ aDepartment of Physiology, School of Medicine, National Yang-Ming University, Taipei, ${ }^{b}$ Cardiovascular \\ Division, Institute of Biomedical Sciences, Academia Sinica, Taipei, 'Division of Cardiovascular Surgery, \\ Department of Surgery, Taipei Veterans General Hospital, Taipei, 'Faculty of Medicine, School of \\ Medicine, National Yang-Ming University, Taipei, e'Graduate Institute and Department of Physiology, \\ College of Medicine, National Taiwan University, Taipei, Taiwan
}

\section{Key Words}

Olanzapine • Hepatic lipid metabolism • Hyperlipidemia • Inflammation • Atherosclerosis

\begin{abstract}
Background/Aims: Olanzapine, an atypical antipsychotic drug, has therapeutic effects for schizophrenia. However, clinical reports indicate that patients taking atypical antipsychotic drugs are at high risk of metabolic syndrome with unclear mechanisms. We investigated the effect of olanzapine on atherosclerosis and the mechanisms in apolipoprotein E-null (apoE${ }^{\prime}$ ) mice. Methods: ApoE ${ }^{-1-}$ mice were used as in vivo models. Western blot analysis was used to evaluate protein expression. Conventional assay kits were applied to assess the levels of cholesterol, triglycerides, free cholesterol, cholesteryl ester, fatty acids, glycerol, and cytokines. Results: Daily treatment with olanzapine (3 $\mathrm{mg} / \mathrm{kg}$ body weight) for four weeks increased mean arterial blood pressure and the whitening of brown adipose tissue in mice. In addition, olanzapine impaired aortic cholesterol homeostasis and exacerbated hyperlipidemia and aortic inflammation, which accelerated atherosclerosis in mice. Moreover, lipid accumulation in liver, particularly total cholesterol, free cholesterol, fatty acids, and glycerol, was increased with olanzapine treatment in apoE $\mathrm{E}^{-/}$mice by upregulating the expression of de novo lipid synthesis-related proteins and downregulating that of cholesterol clearance- or very lowdensity lipoprotein secretion-related proteins. Conclusion: Olanzapine may exacerbate atherosclerosis by deregulating hepatic lipid metabolism and worsening hyperlipidemia and aortic inflammation.




\section{Introduction}

Schizophrenia is a persistent and disabling psychotic disorder that may be attributed to multiple factors including genetic and environmental aspects and social processes $[1,2]$. Schizophrenic patients may display abnormal social behavior and be out of touch with reality $[1,2]$. The most common symptoms of schizophrenia include delusions, hallucinations and disorganized thoughts, which are divided into three categories: positive, negative, and cognitive [3].

The dopamine hypothesis of schizophrenia is the most widely discussed theory in psychiatry and suggests that a disturbed and hyperactive transduction of the dopaminergic signal contributes to schizophrenia symptoms [4, 5]. Targeting this pathway are typical antipsychotic drugs, also called first-generation antipsychotic drugs, which are reported to effectively attenuate positive symptoms of schizophrenia by blocking dopamine $D_{2}$ receptors on postsynaptic neurons [6]. However, because of their high affinity for dopamine $D_{2}$ receptors, typical antipsychotic drugs are associated with a high risk of extrapyramidal motor adverse effects [7].

Recently, atypical antipsychotic agents (second-generation antipsychotic drugs) prescribed for schizophrenia, such as olanzapine and clozapine, were found to incur less risk for extrapyramidal motor side effects because they only partially act as agonists at dopamine $\mathrm{D}_{2}$ and $5-\mathrm{HT}_{1 \mathrm{~A}}$ receptors $[8,9]$. However, evidence has increasingly revealed an association of atypical antipsychotic agent use and the risk of metabolic syndromes including obesity, diabetes, and hyperlipidemia but the mechanism remains unclear [10-12].

The liver is the major organ for both cholesterol production and cholesterol excretion from the human body $[13,14]$. The balance between the delivery of apoB-containing lipoproteins (chylomicron remnants, very low-density lipoprotein [VLDL], intermediatedensity lipoprotein and LDL) from the liver to peripheral tissues and high-density lipoprotein (HDL)-mediated reverse-cholesterol transport from tissues back to the liver is a crucial mechanism for maintaining the appropriate levels of circulating cholesterol [15]. Increased circulating levels of LDL or decreased HDL-mediated cholesterol transport may result in hyperlipidemia, the greatest risk factor for the initiation and progression of atherosclerosis $[16,17]$.

Atherosclerosis is the leading cause of death in developed countries and is characterized by excessive cholesterol deposition and persistent inflammation within the artery wall resulting from the interaction of oxidized LDL, immune cells, and vascular cells $[18,19]$. Evidence accumulating during the past decades suggests that a deregulated inflammatory response and impaired cholesterol metabolism as a result of genetic factors, clinical drug treatment, and environmental toxins accelerate the progression of atherosclerosis [20-22]. However, less is known about the interlocking biology of olanzapine prescribed for schizophrenia and hepatic lipid metabolism or the development of atherosclerosis. Further investigation delineating the effect and molecular mechanisms of olanzapine in the metabolism of cholesterol by the liver and in atherosclerosis is warranted.

Given the impact of atypical antipsychotic agents on the development of metabolic diseases, we aimed to characterize the effect and molecular mechanisms of the use of the atypical antipsychotic drug olanzapine for atherosclerosis. We investigated first the effect of olanzapine on hyperlipidemia, aortic inflammation and the development of atherosclerosis in apolipoprotein E-deficient (apoE ${ }^{-/}$) mice and secondly whether olanzapine affected hepatic lipid metabolism and the possible molecular mechanisms. Olanzapine may deregulate hepatic lipid metabolism, increase hyperlipidemia, and worsen aortic inflammation, ultimately accelerating the development of atherosclerosis. 


\section{Cellular Physiology Cell Physiol Biochem 2018;50:1216-1229 \begin{tabular}{ll|l} 
and Biochemistry & $\begin{array}{l}\text { DOI: 10.1159/000494573 } \\
\text { Published online: 25 October 2018 }\end{array}$ & $\begin{array}{l}\text { (c) } 2018 \text { The Author(s). Published by S. Karger AG, Basel } \\
\text { www.karger.com/cpb }\end{array}$ \\
\hline
\end{tabular} \\ Chen et al.: Olanzapine Exacerbates Atherosclerosis}

\section{Materials and Methods}

\section{Reagents}

Olanzapine was from Cayman Chemical (Ann Arbor, MI, USA). Rabbit antibodies for inducible nitric oxide (iNOS), CD36, scavenger receptor (SR)-BI, 3-hydroxy-3-methyl-glutaryl-coenzyme A reductase (HMGCR), liver X receptor $\beta$ (LXR $\beta$ ), lysosomal acid lipase (LAL), cholesterol 7-alpha-hydroxylase (CYP7A1), ATP-binding cassette sub-family G member 5 (ABCG5), ABCG8, fatty acid synthase (FAS), acetyl-coenzyme A (CoA) carboxylase (ACC), liver-type fatty acid binding protein (L-FABP), diacylglycerol 0-acyltransferase 1 (DGAT1), and long-chain-fatty-acid-CoA ligase 1 (ACSL1), goat antibodies for SR-A, sterol regulatory elementbinding protein 2 (SREBP-2), low-density lipoprotein receptor (LDLR), acyl-CoA:cholesterol acyltransferase 2 (ACAT2), DGAT2, apolipoprotein B (apoB), and microsomal triglyceride transfer protein (MTP), and mouse antibody for SREBP-1 were from Santa Cruz Biotechology (Santa Cruz, CA, USA). Rabbit antibodies for intercellular adhesion molecule 1 (ICAM-1), vascular cell adhesion molecule 1 (VCAM-1) and ABCG1, mouse antibodies for ABCA1, LXR $\alpha$, and carnitine palmitoyltransferase I $\alpha$ (CPT1 $\alpha$, and rat antibody for F4/80 were from Abcam (Cambridge, MA, USA). Mouse antibodies for tubulin and apoAI were from Sigma-Aldrich (St. Louis, MO, USA). Rabbit antibody for LDL receptor-related protein 1 (LRP1) was from Novus (Littleton, CO, USA). ELISA kits for cytokines were from R\&D systems (Minneapolis, MN, USA). Cholesterol and triglyceride assay kits were from Randox (Crumlin, Co. Antrim, UK). Total cholesterol, free cholesterol, cholesteryl ester, triglyceride fatty acid, and glycerol fluorometric assay kits were from BioVision (Milpitas, CA, USA).

\section{Mice}

The investigation conformed to the Guide for the Care and Use of Laboratory Animals (Institute of Laboratory Animal Resources, eighth edition, 2011), and all animal experiments were approved by the Animal Care and Utilization Committee of National Yang-Ming University. ApoE ${ }^{-/}$mice were purchased from Jackson laboratory (Bar Harbor, ME, USA). Mice were housed in barrier facilities, and maintained on a 12-h light/12-h dark cycle. Temperature $\left(22^{\circ} \mathrm{C}\right)$ and humidity $(40-60 \%)$ of the vivarium were tightly controlled. Four to five mice were group-housed per cage and fed a regular chow diet containing $4.5 \%$ fat by weight ( $0.02 \%$ cholesterol) (Newco Distributors, Redwood, CA, USA). Four-month-old male apoE ${ }^{-/-} \mathrm{mice}^{-}$ received daily treatment with olanzapine ( $3 \mathrm{mg} / \mathrm{kg}$ body weight) or vehicle (oil) by gastric gavage for four weeks following previous reports $[23,24]$. At the end of the experiment, the body weight and mean arterial pressure (MAP) of mice were measured and then mice were euthanized with $\mathrm{CO}_{2}$. White (WAT) and brown adipose tissue (BAT) were isolated from the inguinal and gonadal white fat depots and interscapular brown fat depots of mice, respectively. WAT and BAT were weighted and then subjected to histological analysis or stored at $-80{ }^{\circ} \mathrm{C}$. Isolated aortas and livers were homogenized and lysates were subjected to western blot analysis.

\section{Histological examination}

Heart, liver, WAT, and BAT blocks were cut into 8- $\mu \mathrm{m}$ sections and subjected to histological examination. Deparaffinized sections were stained with hematoxylin and eosin (H\&E) and viewed under a Motic TYPE 102M microscope (Motic Images, Xiamen, China). Quantification of the atherosclerotic lesions and content of cellular or non-cellular composition were analyzed by using Motic Images Plus 2.0.

\section{Western blot analysis}

Aortas and livers were lysed in immunoprecipitation $(50 \mathrm{mmol} / \mathrm{L}$ Tris, $\mathrm{pH} \mathrm{7.5,} 5 \mathrm{mmol} / \mathrm{L}$ EDTA, $300 \mathrm{mmol} / \mathrm{L} \mathrm{NaCl}, 1 \%$ Triton X-100, $1 \mathrm{mmol} / \mathrm{L}$ phenylmethylsulfonyl fluoride, $10 \mu \mathrm{g} / \mathrm{mL}$ leupeptin, and $10 \mu \mathrm{g} / \mathrm{mL}$ aprotinin). Aliquots of lysates were separated by sodium dodecyl sulfate polyacrylamide gel electrophoresis (SDS-PAGE) and transblotted onto Immobilon-P membrane, blocked with 5\% skim milk for $1 \mathrm{~h}$ at room temperature, then incubated with primary antibodies overnight followed by the corresponding secondary antibody for $1 \mathrm{~h}$. The protein bands were detected by using an enhanced chemiluminescence kit (PerkinElmer, Boston, MA, USA) and quantified using ImageQuant 5.2 (Healthcare Bio-Sciences, PA, USA). 


\section{Cellular Physiology Cell Physiol Biochem 2018;50:1216-1229

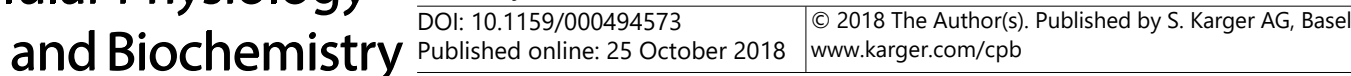 \\ Chen et al.: Olanzapine Exacerbates Atherosclerosis}

\section{Measurement of inflammatory cytokines}

The levels of pro-inflammatory cytokines including tumor necrosis factor- $\alpha$ (TNF- $\alpha$ ), interleukin-1 $\beta$ (IL-1ß), IL-6, monocyte chemoattractant protein-1 (MCP-1), and macrophage inflammatory protein 2 (MIP2) in aortas were measured by using ELISA kits.

\section{Serum lipid profile analysis}

Blood was collected by cardiac puncture. After clotting and centrifugation, serum was isolated and levels of cholesterol, HDL-cholesterol (HDL-c) and triglycerides in serum were measured by using Spotchem EZ SP 4430 (ARKRAY, Inc., Kyoto, Japan).

\section{Determination of hepatic lipids}

The levels of total cholesterol, free cholesterol, cholesteryl ester, triglycerides, fatty acids, and glycerol were measured using fluorescence assay kits (BioVision, Milpitas, CA, USA).

\section{Statistical analysis}

Data are presented as mean \pm SEM. Data from mice were evaluated by parametric tests. The unpaired $t$-test was used to compare two independent groups. SPSS v20.0 (SPSS Inc., Chicago, IL, USA) was used for the analyses. Differences were considered statistically significant at $P<0.05$.

\section{Results}

Effect of olanzapine on body weight, blood pressure and fat tissues in apo $E^{-/ \cdot}$ mice

Previous studies have reported the increased incidence of obesity, hypertension and type2 diabetes; these are all important risk factors for atherosclerosis in schizophrenia patients who take olanzapine [10]. To elucidate the possible effect of olanzapine on atherosclerosis, we used hyperlipidemia- and atherosclerosis-prone apoE $\mathrm{E}^{-/-}$mice as our in vivo model. We first investigated the effect of olanzapine on body weight, blood pressure and adiposity in these mice. Daily treatment with olanzapine for four weeks did not alter body weight but increased MAP as compared with vehicle-treated apoE $/ /$ mice (Fig. 1A, B). Additionally, olanzapine decreased the weight of BAT without affecting that of WAT in apoE ${ }^{-/}$mice (Fig. 1C, D). The number of adipocytes in WAT was increased but their size was decreased in

Fig. 1. Effect of olanzapine on body weight, blood pressure, and fat tissue of apoE $\mathrm{E}^{-/-}$mice. Four-monthsold male apoE $E^{-/} \quad$ mice were orally administered o l a n z a p i n e (OLZ, 3 mg/kg/ day) or vehicle (oil) for four weeks. (A, B) Body weight
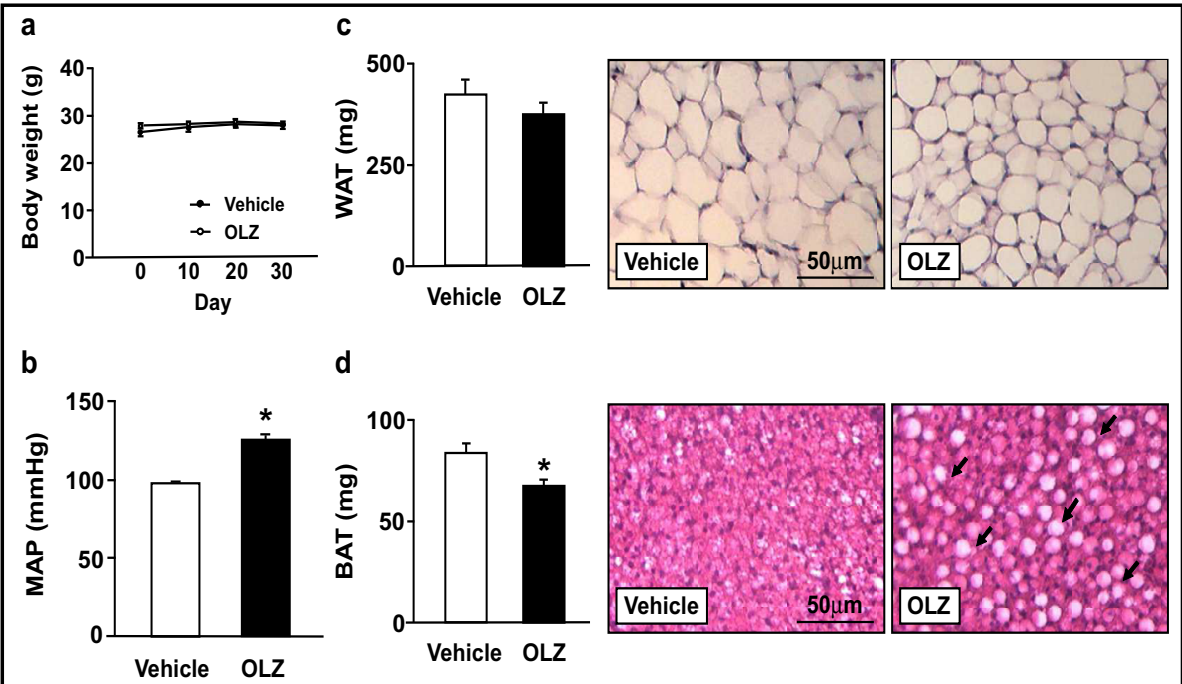

d
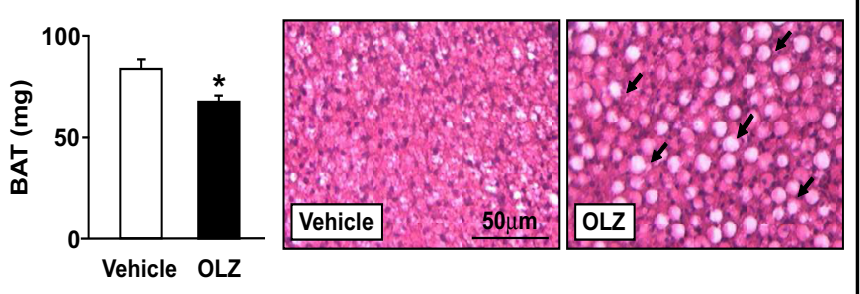

and mean

arterial pressure (MAP). (C, D) Weight and histology of white adipose tissue (WAT) and brown adipose tissue (BAT). Scale bar $=50 \mu \mathrm{m}$. Data are mean \pm SEM from nine mice. ${ }^{*} \mathrm{P}<0.05$ vs. vehicle. Arrows indicate the size of lipid droplets in BAT of OLZ-treated mice. 
olanzapine-treated versus vehicle-treated mice (Fig. 1D). Compared with vehicle treatment, olanzapine significantly increased the size of lipid droplets in BAT (whiting) in apoE $/$ mice (Fig. 1D). These findings suggest that olanzapine may have a detrimental effect on blood pressure and the fat content of adipose tissues.

Fig.

O lan zapine exacerbates atherosclerosis lesion size in apoE $^{-/-}$mice. Four-monthsold male apoE ${ }^{-/} \quad$ mice were orally administered o l a n z a p in e (OLZ, $3 \mathrm{mg} /$ $\mathrm{kg} /$ day) or vehicle (oil) for four weeks. (A)

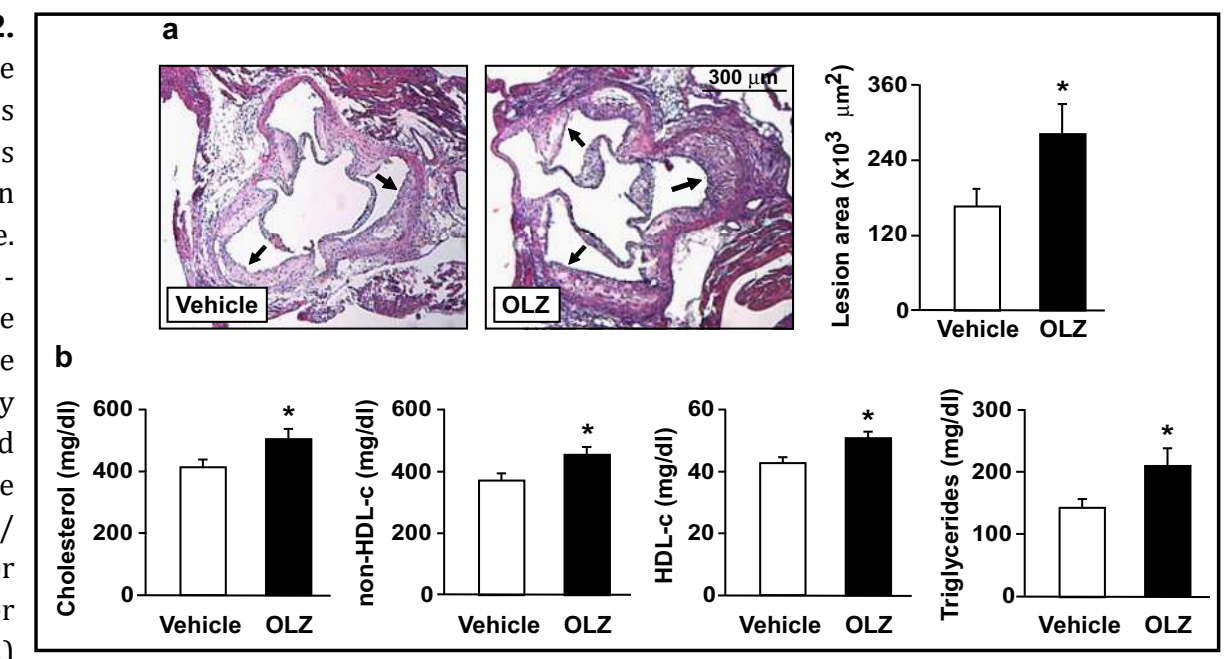

Atherosclerotic

lesions at aortic roots were stained with H\&E. (B) Serum levels of cholesterol, non-high-density lipoprotein cholesterol (non-HDL-c), HDL cholesterol (HDL-c) and triglycerides. Data are mean \pm SEM from nine mice. * $\mathrm{P}<0.05$ vs. vehicle. Scale bar $=300 \mu \mathrm{m}$

Fig. 3. Olanzapine u p r e g u l a t e s scavenger receptors but downregulates reverse cholesterol transporters in apoE-/- aortas. Fourmonths-old male apoE ${ }^{-/}$mice were orally administered olanzapine (OLZ, $3 \mathrm{mg} / \mathrm{kg} /$ day) or vehicle (oil) for four weeks. (A, B) Western blot analysis of protein levels of SR-A, CD36, ABCA1, ABCG1, SR$\mathrm{BI}, \mathrm{LXR} \alpha, \mathrm{LXR} \beta$ and $\alpha$-tubulin in aortas of apoE $\mathrm{E}^{-/-}$mice. Data are mean \pm SEM from nine mice. * $\mathrm{P}<0.05$ vs. vehicle.

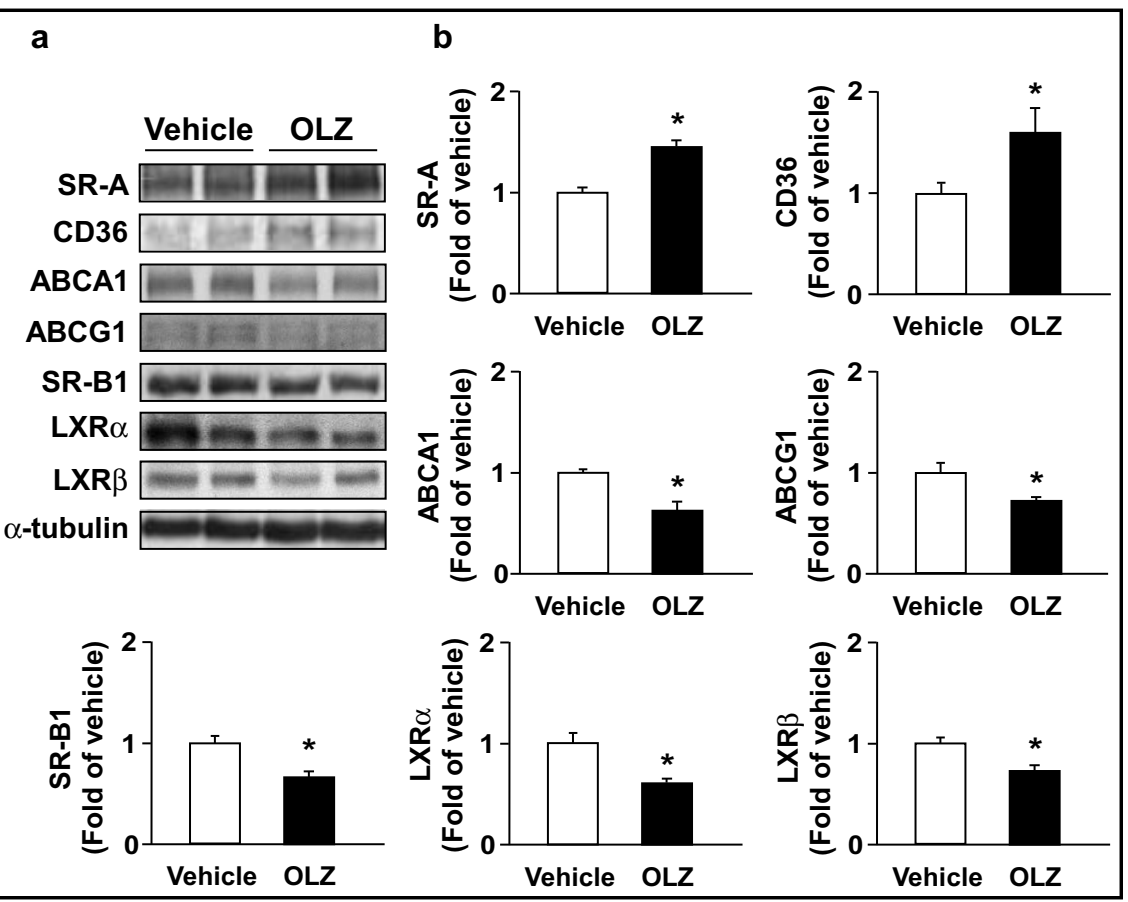




\section{Cellular Physiology Cell Physiol Biochem 2018;50:1216-1229

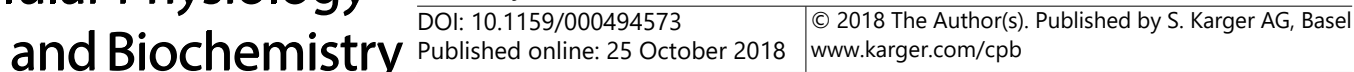

Olanzapine exacerbates atherosclerosis and hyperlipidemia in apo:-/ mice

We next examined the effect of olanzapine on lipoid profile and the progression of atherosclerosis in apo: $\mathrm{E}^{-/}$mice. Compared with vehicle treatment, daily olanzapine treatment greatly increased the size of atherosclerotic lesions at the aortic sinus in apoE $\mathrm{E}^{-/}$mice (Fig. 2A). Furthermore, serum levels of total cholesterol, non-HDL-c, HDL-c and triglycerides were significantly increased in olanzapine-treated apoE $/$ - mice (Fig. 2B), which suggests that olanzapine worsens hyperlipidemia and enhances the development of atherosclerosis.

Olanzapine deregulates cholesterol flux and aggravates inflammation in apoE ${ }^{-/}$mouse aorta

An imbalance in vascular cholesterol flux is crucial for the initiation and progression of atherosclerosis [25]. We therefore examined the effect of olanzapine on the protein expression of scavenger receptors and cholesterol transporters in atherosclerotic aortas of apoE ${ }^{-/}$mice. Treatment with olanzapine significantly upregulated SR-A and CD36 but downregulated ABCA1, ABCG1 and SR-BI as well as LXR $\alpha$ and LXR $\beta$ (Fig. 3A, B), the two key transcript factors for gene regulation of lipid metabolism [26, 27]. Hence, olanzapine may increase cholesterol accumulation in aortas by increasing cholesterol uptake but decreasing cholesterol efflux in atherosclerotic aortas. However, inflammation within atherosclerotic lesions plays a central role in the progression of atherosclerosis $[28,27]$. Therefore, we investigated whether olanzapine promotes the inflammatory response during atherogenesis. Compared with vehicle treatment, olanzapine increased levels of F4/80 (macrophage cell marker), iNOS, VCAM-1, TNF- $\alpha$, IL- 6 and MIP- 2 without changing those of ICAM-1, IL-1 $\beta$ and MCP- 1 in apo- ${ }^{-}$ 1- aortas (Fig. 4A, B). All these are important pro-atherogenic molecules in the development of atherosclerosis [28, 29]. Collectively, olanzapine may aggravate the progression of atherosclerosis by deregulating cholesterol homeostasis and the inflammatory response.

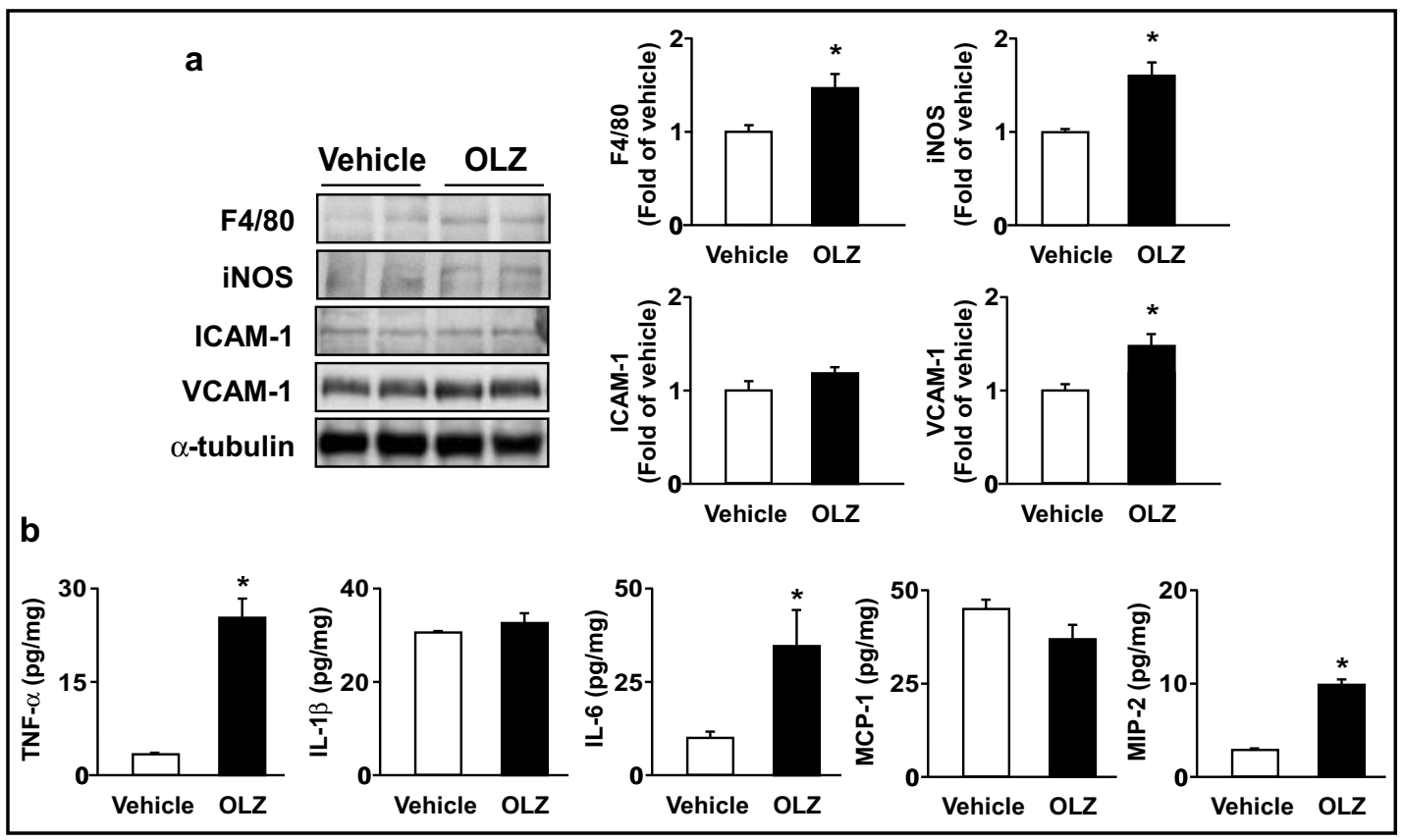

Fig. 4. Olanzapine aggravates inflammation in $\mathrm{apoE}^{-/-}$aortas. Four-months-old male apoE $\mathrm{E}^{-/-}$mice were orally administered olanzapine (OLZ, $3 \mathrm{mg} / \mathrm{kg} /$ day) or vehicle (oil) for four weeks. (A) Western blot analysis of protein levels of F4/80, iNOS, ICAM-1, VCAM-1 and $\alpha$-tubulin in aortas of apoE ${ }^{-/-}$mice. (B) ELISA of aortic levels of TNF- $\alpha$, IL-1 $\beta$, IL-6, MCP-1 and MIP-2. Data are mean \pm SEM from nine mice. ${ }^{*} \mathrm{P}<0.05$ vs. vehicle. 


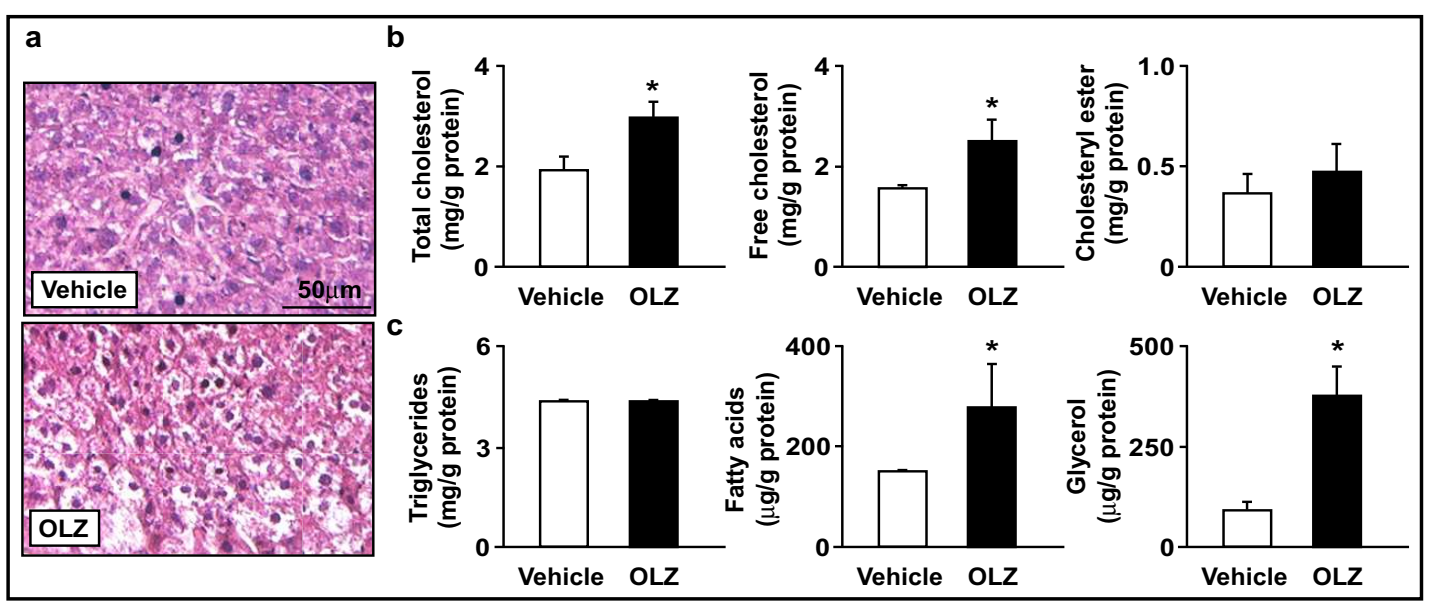

Fig. 5. Olanzapine increases lipid accumulation in apoE $\%$ liver. Four-months-old male apoE ${ }^{-/-}$mice were orally administered olanzapine (OLZ, $3 \mathrm{mg} / \mathrm{kg} / \mathrm{day}$ ) or vehicle (oil) for four weeks. (A) Representative H\&E staining of liver tissue. Scale bar $=50 \mu \mathrm{m}$ (B) Hepatic levels of total cholesterol, free cholesterol and cholesteryl ester. (C) Hepatic levels of triglycerides, fatty acids and glycerol in apoE $/$ mice assessed by fluorometric assay kits. Data are mean \pm SEM from nine mice. ${ }^{*} \mathrm{P}<0.05$ vs. vehicle.

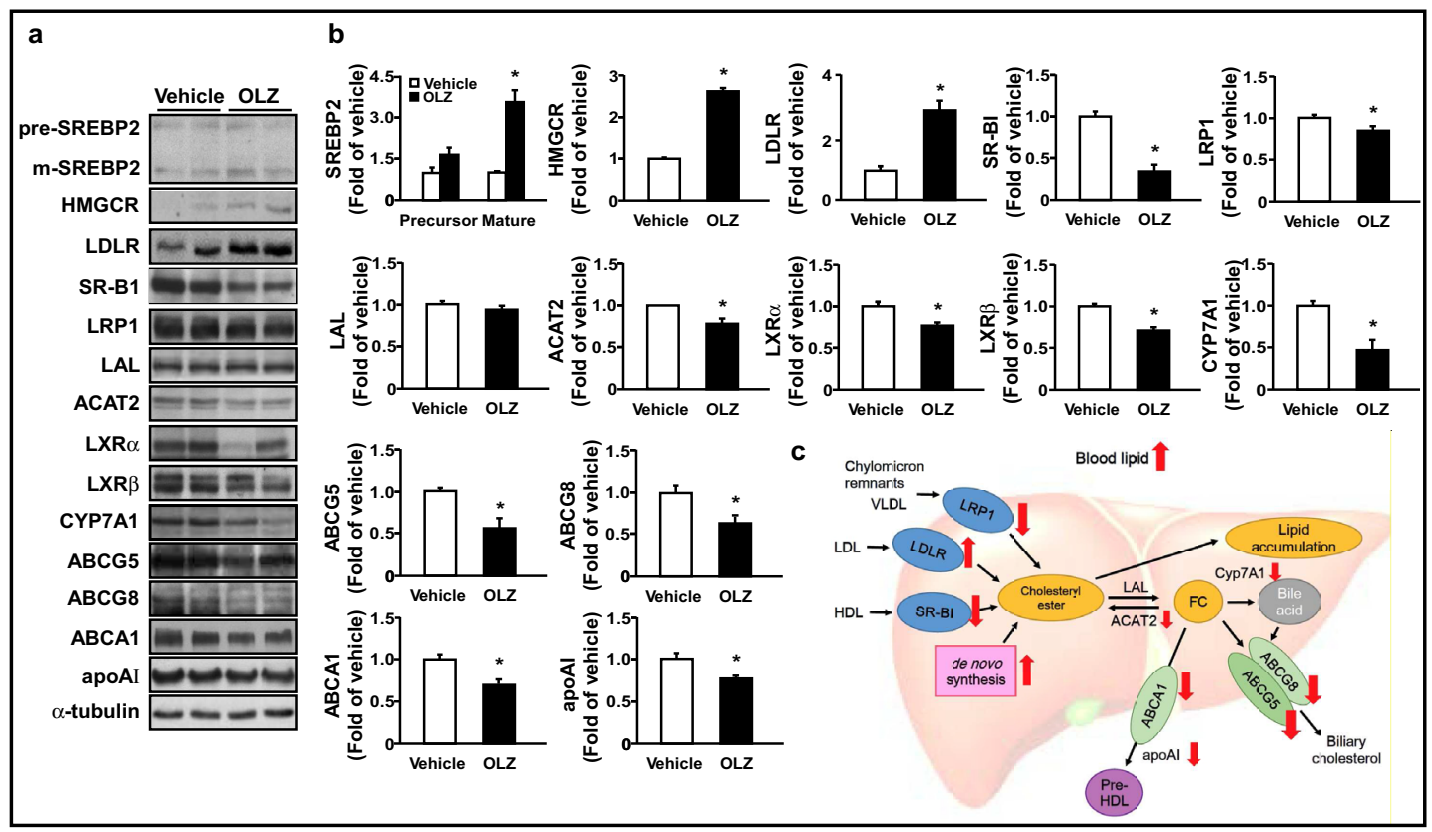

Fig. 6. Effect of olanzapine on de novo cholesterol synthesis-, lipoprotein metabolism-, bile acid metabolism-, and cholesterol efflux-related protein expression in apoE $\%$ liver. Four-months-old male apoE $/$ mice were orally administered olanzapine (OLZ, $3 \mathrm{mg} / \mathrm{kg} /$ day) or vehicle (oil) for four weeks. (A, B) Western blot analysis of protein levels of SREBP2, HMGCR, LDLR, SR-BI, LDLR, LRP1, LAL, ACAT2, LXR LXR $\beta$, CYP7A1, ABCG5, ABCG8, ABCA1, apoAI and $\alpha$-tubulin in the liver of apoE ${ }^{-/-}$mice. Data are mean \pm SEM from nine mice. ${ }^{*} \mathrm{P}<0.05$ vs. vehicle.

\section{Olanzapine increases lipid accumulation in apoE ${ }^{-/}$mouse liver}

The liver is the major organ for lipid metabolism in the human body [30]. Disruption of hepatic lipid homeostasis leads to lipid accumulation in the liver or hyperlipidemia [31]. We examined the effect of olanzapine on hepatic lipid metabolism and the potential mechanism(s). Daily treatment with olanzapine for four weeks increased the hepatic levels of total cholesterol, free cholesterol, fatty acids and glycerol but not of cholesteryl ester and triglycerides in apoE $\mathrm{E}^{-/-}$mouse liver (Fig. 5A-C). 


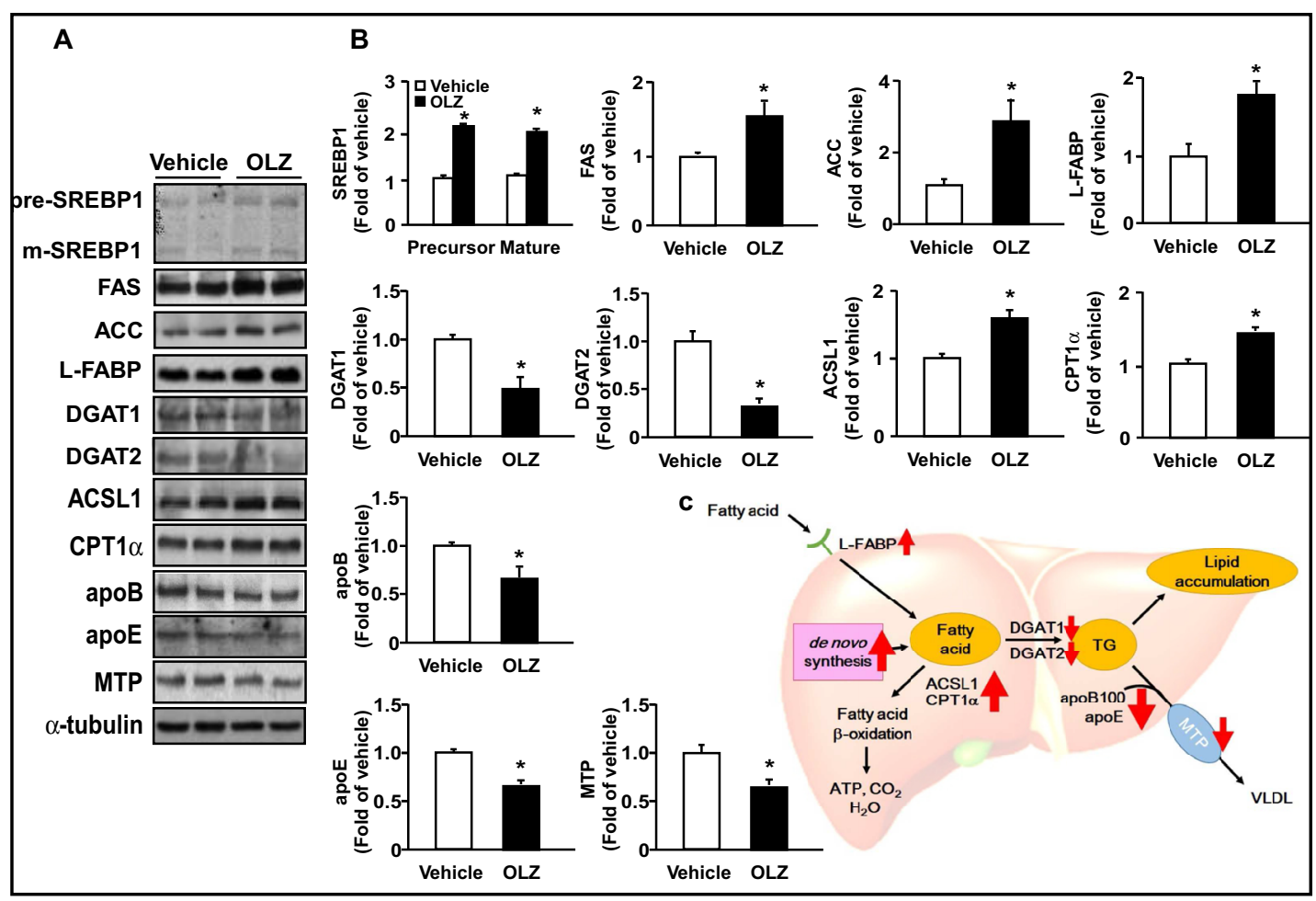

Fig. 7. Effect of olanzapine on de novo fatty acid synthesis-, triglyceride synthesis-, $\beta$-oxidation of fatty acids- and VLDL secretion-related protein expression in apoE $E^{-/-}$liver. Four-months-old male apoE $\mathrm{E}^{-/-}$mice were orally administered olanzapine (OLZ, $3 \mathrm{mg} / \mathrm{kg} /$ day) or vehicle (oil) for four weeks. (A, B) Western blot analysis of protein levels of SREBP1, FAS, ACC, L-FABP, DGAT1, DGAT2, ACSL1, CPT1 $\alpha$, apoB, apoE, MTP and $\alpha$-tubulin in the liver of apoE $\mathrm{E}^{-/-}$mice. Data are mean \pm SEM from nine mice. ${ }^{*} \mathrm{P}<0.05$ vs. vehicle.

Olanzapine deregulates hepatic cholesterol and triglyceride metabolism in apoE ${ }^{-1-}$ mice

Hepatic cholesterol and triglyceride metabolism are crucial factors for maintaining the homeostasis of the lipid pool $[13,14]$. Thus, the effects of olanzapine on hepatic de novo lipogenesis, lipoprotein metabolism, triglyceride synthesis, and secretion, as well as fatty acid oxidation were investigated. We demonstrated that olanzapine increased the expression of the de novo cholesterol synthesis-related proteins SREBP2 and HMGCR, and of LDLR, the receptor for LDL internalization, but decreased that of SR-BI and LRP-1, the receptors for HDL or chylomicron remnants and VLDL uptake, respectively [32]. Moreover, the expression of cholesterol esterification- and clearance-related proteins including ACAT2, LXR $\alpha$, LXR $\beta$, CYP7A1, ABCG5, ABCG8, ABCA1, and apoAI was decreased with olanzapine treatment (Fig. $6 \mathrm{~A}, \mathrm{~B})$. These results suggest that olanzapine upregulates de novo synthase-related proteins and downregulates esterification- and clearance-related proteins, thereby leading to the accumulation of cholesterol in the liver (Fig. 6C).

We further examined whether triglyceride metabolism was affected by olanzapine. Olanzapine upregulated de novo fatty acid synthesis-related proteins SREBP1, ACC and FAS but downregulated the triglyceride synthesis-related proteins DGAT1 and DGAT2 (Fig. 7A, B). Furthermore, VLDL secretion-related proteins, including apoB100, apoE and MTP were downregulated with olanzapine treatment. The expression of proteins associated with $\beta$-oxidation, such as ACSL1 and CPT1 $\alpha$ was increased with olanzapine treatment (Fig. 7A, B). As a whole, the data suggest that olanzapine increases de novo synthesis of fatty acids but decreases the conversion of fatty acid to triglycerides, leading to the elevation of fatty acid in the liver, ultimately increasing $\beta$-oxidation and decreasing VLDL secretion (Fig. 7C).

Collectively, daily olanzapine treatment exacerbates aortic inflammation, aggravated 


\section{Cellular Physiology Cell Physiol Biochem 2018;50:1216-1229

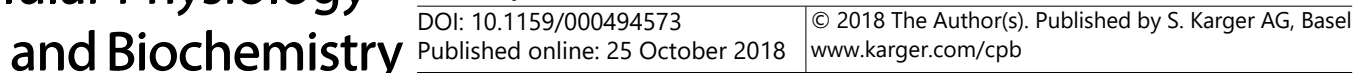

hyperlipidemia, deregulated hepatic lipid metabolism and, ultimately, worsens atherosclerosis in $\mathrm{apoE}^{-/}$ mice (Fig. 8).

\section{Discussion}

Patients with schizophrenia who are taking atypical antipsychotic agents are known to be at high risk for metabolic syndrome, a well-known risk factor for atherosclerosis and related cardiovascular diseases [1012]. However, the effect of atypical antipsychotic agents on atherogenesis remains elusive. In this study, we

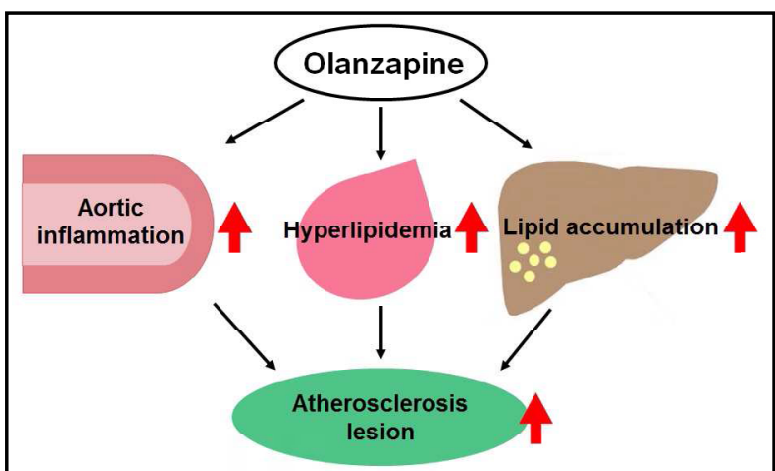

Fig. 8. Proposed model of mechanisms by which olanzapine accelerates the progression to atherosclerosis. Olanzapine exacerbates aortic inflammation, aggravates hyperlipidemia and deregulates hepatic lipid metabolism of apoE $\mathrm{E}^{-/}$mice, thereby accelerating atherosclerosis. provide new insights into the effect of an atypical antipsychotic agent, olanzapine, on the pathogenesis of atherosclerosis and the underlying molecular mechanism. Chronic treatment with olanzapine for four weeks deregulated hepatic lipid metabolism and exacerbated hyperlipidemia and aortic inflammation of apoE $\mathrm{E}^{\%}$ mice, ultimately aggravating atherosclerosis. The use of atypical antipsychotic agents in schizophrenia may have detrimental effects on the development of atherosclerosis.

However, our knowledge about the molecular mechanism behind the pro-atherogenic effect of olanzapine is less detailed. We used a hyperlipidemia mouse model to investigate the mechanism. We found that olanzapine could deregulate the adiposity of BAT as evidenced by the increased whiting of BAT, which agreed with previous findings that olanzapine upregulated aP2 expression, the marker of WAT adipocytes, but downregulated UCP1 expression, the marker of BAT adipocytes, in adipose tissues of rats $[33,34]$. To date, the detailed mechanism underlying the whiting of BAT is not fully understood. Recently, Shimizu et al. reported that the phenotype of BAT whiting in mice is characterized by mitochondrial dysfunction, lipid accumulation, and downregulation of vascular endothelial growth factor, and might be attributed to obesity-induced capillary rarefaction and hypoxia in BAT [35]. However, how olanzapine induces the whiting of BAT is still elusive. Further investigation delineating the effect and molecular mechanisms of olanzapine in the BAT is warranted.

Intriguingly, treatment with olanzapine did not affect the body weight of apo $\mathrm{E}^{-/}$mice within our four-week experimental period, which, however, was inconsistent with the previous findings reporting that treatment with olanzapine increased the body weight of human patients and experimental rats over longer experimental periods [34, 36]. Our data agree with Albaugh et al. and Shertzer et al., who found that treatment with olanzapine did not alter body weight in the mouse model $[37,38]$. Although the exact mechanism is unclear, the reason for the discrepancies between our study and previous studies may be the difference in species or the genetic background of experimental animals or the length of the experimental period.

Besides the pro-obesity effect of olanzapine, we also found that it increased the MAP of apoE $/$ mice, which agrees with clinical observations that patients under olanzapine or clozapine treatment run an increased risk of hypertension [12]. Although the exact mechanism is not known, hypertension is considered one of the major risk factors for the development of atherosclerosis in humans $[39,40]$. Angiotensin II, the major player in hypertension, increases oxidative stress, induces endothelial dysfunction and promotes the proliferation and migration of smooth muscle cells, thereby leading to the progression of atherosclerotic lesions in experimental animal studies [41, 42]. However, whether the increased blood pressure induced by olanzapine contributes to the exacerbation of atherosclerosis and its 


\section{Cellular Physiology Cell Physiol Biochem 2018;50:1216-1229 \begin{tabular}{ll|l} 
DOI: 10.1159/000494573 & O 2018 The Author(s). Published by S. Karger AG, Basel \\
www.karger.com/cpb
\end{tabular} \\ Chen et al.: Olanzapine Exacerbates Atherosclerosis}

underlying molecular mechanism(s) remains unclear. Further studies investigating the molecular mechanism underlying the hypertensive effect of olanzapine are warranted.

With olanzapine treatment, the hyperlipidemia of apoE $^{-/-}$mice was exacerbated as evidenced by the increases in serum levels of total cholesterol, non-HDL-c, HDL-c and triglycerides. It is well established that dietary fats and those from de novo lipogenesis in the liver are the two main sources for circulating total cholesterol and triglycerides, which are carried in circulation as VLDL. Elevated circulating total cholesterol and triglycerides may be attributed to increased lipid synthesis, or decreased lipid clearance, or both in the liver [13-17]. In this study, no difference was found in food intake between vehicle-treated mice and olanzapine-treated mice (data not shown). Our findings demonstrated that olanzapine significantly decreased the protein expression of LRP1, the receptor for VLDL and chylomicron remnants in the liver, suggesting that impaired LRP1-mediated lipid clearance may be one of main causes for higher plasma total cholesterol and triglycerides in olanzapine-treated mice.

Deregulation of the macrophage-mediated cholesterol metabolism and inflammation in the artery wall is crucial in the initiation and progression of atherosclerosis [25]. Mainly, excess cholesterol accumulated in macrophages is primarily due to uncontrolled uptake of oxidized LDL or impaired cholesterol efflux [25]. We found that olanzapine upregulated the expression of SR-A and CD36 but downregulated that of ABCA1, ABCG1 and SR-BI. In addition to the critical effect of hyperlipidemia in the initiation of atherosclerosis, the progression of atherosclerotic lesions is determined by the inflammatory response within the artery wall $[28,29]$. Interestingly, our results showed that olanzapine downregulated the aortic expression of ABCA1/ABCG1, 2 important regulators for cholesterol efflux from peripheral tissues to HDL or apo A-I [25-27]. Theoretically, the plasma level of HDL-c should be lower in olanzapine-treated mice than in vehicle-treated mice. However, our results also showed that olanzapine markedly decreased the hepatic level of SR-BI protein, the HDL receptor for the selective uptake of cholesterol ester from circulating HDL, and thus promoted the excretion of cholesterol into bile, leading to the elimination of excess cholesterol from the human body $[26,27]$. We thus thought that the increase in plasma HDL-c driven by olanzapine might be attributed to the impairment of the SR-BI-mediated uptake of cholesterol ester from HDL.

Notably, we demonstrated that olanzapine promoted aortic inflammation, as evidenced by increased levels of F4/80 (a cell marker of macrophage), iNOS, VCAM-1, TNF- $\alpha$, IL-6 and MIP-2 in aortic lysates, albeit inconsistent with the findings by Sugino et al., who reported that atypical antipsychotics including olanzapine suppressed lipopolysaccharide-induced acute systemic inflammation in mice [43]. Our results suggest that the local inflammatory response within atherosclerotic lesions rather than systemic inflammation plays an important role in the olanzapine-induced progression of atherosclerosis in $\mathrm{apoE}^{-/-}$mice. One possible explanation for the discrepancy between our study and previous research may be the difference in murine disease models. In view of the function of these proteins, olanzapine may disturb cholesterol homeostasis and regulation of the inflammatory response, thereby accelerating atherosclerosis in apoE ${ }^{-/}$mice. Despite our unique findings, the detailed mechanisms by which olanzapine modulates lipid metabolism and the inflammatory response within atherosclerotic lesions merit further study.

Liver-mediated lipid metabolism plays a central role in controlling whole-body lipid homeostasis, including cholesterol and triglycerides with fluxes in dietary, circulating and peripheral local lipid pools $[32,44]$. Under physiological conditions, the lipid content of liver is tightly controlled by the integration of several aspects of metabolic pathways, including de novo lipogenesis, $\beta$-oxidation of fatty acids, VLDL synthesis, lipoprotein internalization from circulation and bile acid synthesis [45-48]. Our findings that treatment with olanzapine significantly increased the lipid content of the liver, particularly total cholesterol, free cholesterol, fatty acids, and glycerol, suggested the unfavorable effect of olanzapine on hepatic lipid metabolism in hyperlipidemic apoE $\mathrm{E}^{-/}$mice. This observation agrees with previous reports of atypical antipsychotic agents disturbing hepatic lipid metabolism [48]. Nevertheless, the potential mechanism underlying the deregulation of lipid homeostasis by atypical antipsychotic agents remains poorly understood. 


\section{Cellular Physiology Cell Physiol Biochem 2018;50:1216-1229

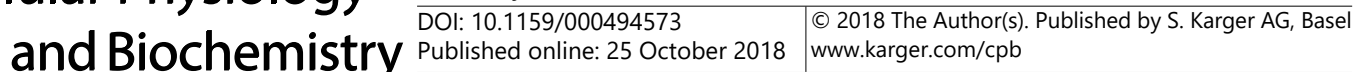

We subsequently asked how olanzapine affected hepatic lipid metabolism. Olanzapine activated SREBP-1 and -2 and the expression of FAS, ACC, HMGCR and LDLR, all crucial regulators in the de novo synthesis of hepatic triglycerides and cholesterol [26, 32]. Moreover, olanzapine decreased the uptake of lipoproteins from circulation, as evidenced by the downregulation of SR-BI, or LRP1, which are HDL or chylomicron remnants and VLDL receptors, respectively [32]. This deregulation of the lipoprotein metabolism may contribute to the elevated levels of circulating cholesterol and triglycerides. More importantly, olanzapine decreased the protein expression of LXR $\alpha$, apoAI, ABCA1, CYP7A1, ABCG5 and ABCG8, all implicated in hepatic cholesterol efflux and bile acid metabolism [32, 49]. In terms of function, the downregulation of these proteins by olanzapine is likely one of the mechanisms for the olanzapine-increased accumulation of lipids in the liver. Notably, the protein expression of ACSL1 and CPT- $1 \alpha$, two key regulators in the $\beta$-oxidation of fatty acids $[50,51]$, was increased. Although the detailed mechanism was unclear, this response may be a compensatory mechanism for the olanzapine-increased fatty acid accumulation in the liver. Moreover, we found that the elevated free cholesterol could be toxic to cells in the liver as shown by increased numbers of apoptotic cells in the livers of olanzapinetreated mice (data not shown). Overall, congruous data are observed in the alteration of key molecules regulating lipid metabolism, which demonstrates again the detrimental effects of olanzapine on hepatic lipid metabolism. The disturbed hepatic lipid metabolism appeared to be the key event for the olanzapine-induced exacerbation of hyperlipidemia and subsequent progression of atherosclerosis in apo: $\mathrm{E}^{-/}$mice.

Nevertheless, our study contains several limitations because we did not use a schizophrenia animal model for experimental analysis. A specific schizophrenia mouse model with hyperlipidemia is not available, so we could not clarify the effects of atypical antipsychotic agents on lipid homeostasis under these pathological conditions. In addition, the cardiovascular significance of atypical antipsychotic agents in translational or clinical medicine is not fully clarified. We do not have clinical data to support our observations from animal studies. To this end, further investigations describing the implications of atypical antipsychotic agents for hepatic lipid metabolism and cardiovascular diseases are warranted.

Atherosclerosis is a complex, progressive disease process with multiple etiologies [18-22]. It is well established that hypertension, hyperlipidemia, and inflammation are crucial risk factors for the development of atherosclerosis $[25,28,29,40]$. In this study, we found that olanzapine increases mean arterial pressure, exacerbates hyperlipidemia, and deregulates aortic inflammation and cholesterol metabolism; all of these events induced by olanzapine could accelerate the progression of atherosclerosis.

\section{Conclusion}

In summary, our study provides advanced evidence that olanzapine has multifaceted effects on hepatic lipid metabolism by increasing de novo lipogenesis, by decreasing lipoprotein internalization, cholesterol clearance, and efflux, and by reducing VLDL secretion, which exacerbate lipid accumulation in the liver and hyperlipidemia as well as the progression of atherosclerosis. Also, we discovered a link between atypical antipsychotic agents, hepatic lipid metabolism and cardiovascular biology, which broadens the clinical implications of atypical antipsychotic agents for the development of cardiovascular diseases.

\section{Acknowledgements}

The authors thank Laura Smales for help in language editing. This study was supported by grants from the Ministry of Science and Technology of Taiwan (MOST- 103-2320-B-010040-MY3, 105-2320-B-010-036, 105-2811-B-010-022 and 106-2320-B-002-05) and Taipei Veterans General Hospital (V105D18-003-MY3-3), Taiwan. 


\section{Cellular Physiology Cell Physiol Biochem 2018;50:1216-1229 \begin{tabular}{ll|l} 
and Biochemistry & $\begin{array}{l}\text { DOI: } 10.1159 / 000494573 \\
\text { Published online: } 25 \text { October } 2018\end{array}$ & $\begin{array}{l}\text { (c) } 2018 \text { The Author(s). Published by S. Karger AG, Basel } \\
\text { www.karger.com/cpb }\end{array}$
\end{tabular} \\ Chen et al.: Olanzapine Exacerbates Atherosclerosis}

\section{Disclosure Statement}

The authors have no conflicts of interest to declare.

\section{References}

1 Maksymetz J, Moran SP, Conn PJ: Targeting metabotropic glutamate receptors for novel treatments of schizophrenia. Mol Brain 2017;10:15.

2 Najjar S, Pahlajani S, De Sanctis V, Stern JNH, Najjar A, Chong D: Neurovascular unit dysfunction and bloodbrain barrier hyperpermeability contribute to schizophrenia neurobiology: A theoretical integration of clinical and experimental evidence. Front Psychiatry 2017;8:83.

3 Harvey PD, Koren D, Reichenberg A, Bowie CR: Negative symptoms and cognitive deficits: what is the nature of their relationship? Schizophr Bull 2006;32:250-258.

$\checkmark 4$ Lodge DJ, Grace AA: Hippocampal dysregulation of dopamine system function and the pathology of schizophrenia. Trends Pharmacol Sci 2011;32:507-513.

5 Perez-Costas E, Melendez-Ferro M, Roberts RC: Basal ganglia pathology in schizophrenia: dopamine connections and anomalies. J Neurochem 2010;113:287-302.

6 Li P, Snyder GL, Vanover KE: Dopamine targeting drugs for the treatment of schizophrenia: past, present and future. Curr Top Med Chem 2016;16:3385-3403.

7 Sykes DA, Moore H, Stott L, Holliday N, Javitch JA, Lane JR, Charlton SJ: Extrapyramidal side effects of antipsychotics are linked to their association kinetics at dopamine D2 receptors. Nat Commun 2017;8:763.

8 Haleem DJ: 5-HT1A receptor-dependent control of nigrostriatal dopamine neurotransmission in the pharmacotherapy of Parkinson's disease and schizophremia. Brhay Pharmacol 2015;26:45-58.

-9 Kinon BJ, Lieberman JA: Mechanisms of action of atypical antipsychotic drugs: a critical analysis. Psychopharmacology (Berl) 1996;124:2-34.

10 Glowinska B, Urban M, Koput A, Galar M: New atherosclerosis risk factors in obese, hypertensive and diabetic children and adolescents. Atherosclerosis 2003;167:275-286.

11 Koro CE, Meyer JM: Atypical antipsychotic therapy and hyperlipidemia: a review. Essent Psychopharmacol 2005;6:148-157.

>12 Hirsch L, Yang J, Bresee L, Jette N, Patten S, Pringsheim T: Second-generation antipsychotics and metabolic side effects: A systematic review of population-based studies. Drug Saf 2017;40:771-781.

13 de Guia RM, Rose AJ, Herzig S: Glucocorticoid hormones and energy homeostasis. Horm Mol Biol Clin Investig 2014;19:117-128.

14 Nemes K, Åberg F, Gylling H, Isoniemi H: Cholesterol metabolism in cholestatic liver disease and liver transplantation: from molecular mechanisms to clinical implications. World J Hepatol 2016;8:924-932.

-15 März W, Kleber ME, Scharnagl H, Speer T, Zewinger S, Ritsch A, Parhofer KG, von Eckardstein A, Landmesser U, Laufs U: HDL cholesterol: reappraisal of its clinical relevance. Clin Res Cardiol 2017;106:663-675.

16 Badimon L, Vilahur G: LDL-cholesterol versus HDL-cholesterol in the atherosclerotic plaque: inflammatory resolution versus thrombotic chaos. Ann N Y Acad Sci 2012;1254:18-32.

17 Libby P: Managing the risk of atherosclerosis: the role of high-density lipoprotein. Am J Cardiol 2001;88:3N-8N.

18 Li AC, Glass CK: The macrophage foam cell as a target for therapeutic intervention. Nat Med 2002;8:12351242.

19 Matsuura E, Kobayashi K, Tabuchi M, Lopez LR: Oxidative modification of low-density lipoprotein and immune regulation of atherosclerosis. Prog Lipid Res 2006;45:466-486.

-20 Rader DJ, Daugherty A: Translating molecular discoveries into new therapies for atherosclerosis. Nature 2008;451:904-913.

-21 Upadhyay RK: Emerging risk biomarkers in cardiovascular diseases and disorders. J Lipids 2015;2015: 971453.

22 Zhao JF, Hsiao SH, Hsu MH, Pao KC, Kou YR, Shyue SK, Lee TS: Di-(2-ethylhexyl) phthalate accelerates atherosclerosis in apolipoprotein E-deficient mice. Arch Toxicol 2016;90:181-190. 


\section{Cellular Physiology Cell Physiol Biochem 2018;50:1216-1229 \begin{tabular}{ll|l} 
DOl: 10.1159/000494573 & $\begin{array}{l}\text { O 2018 The Author(s). Published by S. Karger AG, Basel } \\
\text { www.karger.com/cpb }\end{array}$ \\
\hline
\end{tabular} \\ Chen et al.: Olanzapine Exacerbates Atherosclerosis}

-23 Coccurello R1, Caprioli A, Conti R, Ghirardi O, Borsini F, Carminati P, Moles A: Olanzapine (LY170053, 2-methyl-4-(4-methyl-1-piperazinyl)-10H-thieno [2, 3-b] [1, 5] benzodiazepine), but not the novel atypical antipsychotic ST2472 (9-piperazin-1-ylpyrrolo [2, 1-b][1, 3]benzothiazepine), chronic administration induces weight gain, hyperphagia, and metabolic dysregulation in mice. J Pharmacol Exp Ther 2008;326:905-911.

-24 Shertzer HG1, Kendig EL, Nasrallah HA, Johansson E, Genter MB: Protection from olanzapine-induced metabolic toxicity in mice by acetaminophen and tetrahydroindenoindole. Int J Obes (Lond) 2010;34:970979.

25 Zhao JF, Ching LC, Huang YC, Chen CY, Chiang AN, Kou YR, Shyue SK, Lee TS: Molecular mechanism of curcumin on the suppression of cholesterol accumulation in macrophage foam cells and atherosclerosis. Mol Nutr Food Res 2012;56:691-701.

-26 Ulven SM, Dalen KT, Gustafsson JA, Nebb HI: LXR is crucial in lipid metabolism. Prostaglandins Leukot Essent Fatty Acids 2005;73:59-63.

-27 Wójcicka G, Jamroz-Wiśniewska A, Horoszewicz K, Bełtowski J: Liver X receptors (LXRs). Part I: structure, function, regulation of activity, and role in lipid metabolism. Postepy Hig Med Dosw (Online) 2007;61:736759.

28 Galkina E, Ley K: Immune and inflammatory mechanisms of atherosclerosis. Annu Rev Immunol 2009;27:165-197.

-29 Kuziel WA, Dawson TC, Quinones M, Garavito E, Chenaux G, Ahuja SS, Reddick RL, Maeda N: CCR5 deficiency is not protective in the early stages of atherogenesis in apoE knockout mice. Atherosclerosis. 2003;167:25-32.

30 Rui L: Energy metabolism in the liver. Compr Physiol 2014;4:177-197.

-31 Bechmann LP, Hannivoort RA, Gerken G, Hotamisligil GS, Trauner M, Canbay A: The interaction of hepatic lipid and glucose metabolism in liver diseases. J Hepatol 2012;56:952-964.

-32 Kiss RS, Sniderman A: Shunts, channels and lipoprotein endosomal traffic: a new model of cholesterol homeostasis in the hepatocyte. J Biomed Res 2017;31:95-107.

33 Zhang Q, Lian J, He M, Deng C, Wang H, Huang XF: Olanzapine reduced brown adipose tissue thermogenesis and locomotor activity in female rats. Prog Neuropsychopharmacol Biol Psychiatry 2014;51:172-180.

-34 Lian J, Huang XF, Pai N, Deng C: Preventing olanzapine-induced weight gain using betahistine: a study in a rat model with chronic olanzapine treatment. PLoS One 2014;9:e104160.

-35 Shimizu I, Aprahamian T, Kikuchi R, Shimizu A, Papanicolaou KN, MacLauchlan S, Maruyama S, Walsh K: Vascular rarefaction mediates whitening of brown fat in obesity. J Clin Invest 2014;124:2099-2112.

36 Goudie AJ, Cooper GD, Halford JC: Antipsychotic-induced weight gain. Diabetes Obes Metab 2005;7:478487.

-37 Albaugh VL, Henry CR, Bello NT, Hajnal A, Lynch SL, Halle B, Lynch CJ: Hormonal and metabolic effects of olanzapine and clozapine related to body weight in rodents. Obesity (Silver Spring) 2006;14:36-51.

-38 Shertzer HG, Kendig EL, Nasrallah HA, Johansson E, Genter MB: Protection from olanzapine-induced metabolic toxicity in mice by acetaminophen and tetrahydroindenoindole. Int J Obes (Lond) 2010;34:970979.

39 Kobayashi M, Uesugi S: The role of hypertension as a risk factor of atherosclerosis. Rinsho Byori 1995;43:104-110.

40 Rafieian-Kopaei M, Setorki M, Doudi M, Baradaran A, Nasri H: Atherosclerosis: process, indicators, risk factors and new hopes. Int J Prev Med 2014;5:927-946.

41 Gomolak JR, Didion SP: Angiotensin II-induced endothelial dysfunction is temporally linked with increases in interleukin-6 and vascular macrophage accumulation. Front Physiol 2014;5:396.

-42 Milliat F, François A, Isoir M, Deutsch E, Tamarat R, Tarlet G, Atfi A, Validire P, Bourhis J, Sabourin JC, Benderitter M: Influence of endothelial cells on vascular smooth muscle cells phenotype after irradiation. Am J Pathol 2006;169:1484-1495.

43 Sugino H, Futamura T, Mitsumoto Y, Maeda K, Marunaka Y: Atypical antipsychotics suppress production of proinflammatory cytokines and up-regulate interleukin-10 in lipopolysaccharide-treated mice. Prog Neuropsychopharmacol Biol Psychiatry 2009;33:303-307.

$\$ 44$ Trapani L, Segatto M, Pallottini V: Regulation and deregulation of cholesterol homeostasis: the liver as a metabolic "power station". World J Hepatol 2012;4:184-190. 


\section{Cellular Physiology Cell Physiol Biochem 2018;50:1216-1229

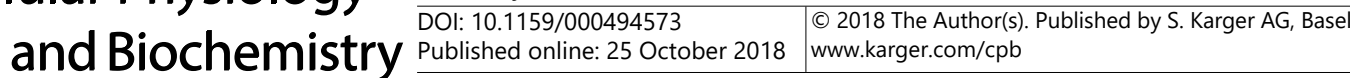 \\ Chen et al.: Olanzapine Exacerbates Atherosclerosis}

-45 Chiang JY: Bile acid metabolism and signaling. Compr Physiol 2013;3:1191-1212.

46 Sanders FWB, Griffin JL: De novo lipogenesis in the liver in health and disease: more than just a shunting yard for glucose. Biol Rev Camb Philos Soc 2016;91:452-468.

47 Singh H, Derwas N, Poulos A: Very long chain fatty acid beta-oxidation by rat liver mitochondria and peroxisomes. Arch Biochem Biophys 1987;259:382-390.

-48 Koponen H, Saari K, Savolainen M, Isohanni M: Weight gain and glucose and lipid metabolism disturbances during antipsychotic medication: a review. Eur Arch Psychiatry Clin Neurosci 2002;252:294-298.

-49 Zhu R, Ou Z, Ruan X, Gong J: Role of liver X receptors in cholesterol efflux and inflammatory signaling. Mol Med Rep 2012;5:895-900.

50 Ellis JM, Li LO, Wu PC, Koves TR, Ilkayeva O, Stevens RD, Watkins SM, Muoio DM, Coleman RA: Adipose acylCoA synthetase-1 (ACSL1) directs fatty acids towards $\beta$-oxidation and is required for cold thermogenesis. Cell Metab 2010;12:53-64.

51 Foster DW: Malonyl-CoA: the regulator of fatty acid synthesis and oxidation. J Clin Invest 2012;122:19581959. 